\title{
MULTIPLE POSITIVE SOLUTIONS FOR A NONLINEAR CHOQUARD EQUATION WITH NONHOMOGENEOUS
}

\author{
Hong-Yao Li, Chun-Lei TANG And Xing-Ping Wu
}

Abstract. In this paper, we study the existence of multiple positive solutions for the following equation:

$$
-\Delta u+u=\left(K_{\alpha}(x) *|u|^{p}\right)|u|^{p-2} u+\lambda f(x), \quad x \in \mathbb{R}^{N},
$$

where $N \geqslant 3, \alpha \in(0, N), p \in(1+\alpha / N,(N+\alpha) /(N-2)), K_{\alpha}(x)$ is the Riesz potential, and $f(x) \in H^{-1}\left(\mathbb{R}^{N}\right), f(x) \geqslant 0, f(x) \not \equiv 0$. We prove that there exists a constant $\lambda^{*}>0$ such that the equation above possesses at least two positive solutions for all $\lambda \in\left(0, \lambda^{*}\right)$. Furthermore, we can obtain the existence of the ground state solution.

Mathematics subject classification (2010): 26D15, 26A51, 32F99, 41A17.

Keywords and phrases: Positive solutions, nonlinear Choquard's equation, mountain pass lemma, Ekeland's variational principle.

\section{REFERENCES}

[1] E. H. Lieb And B. Simon, The Hartree-Fock theory for Coulomb systems, Comm. Math. Phys. 53, 3 (1977), 185-194.

[2] E. H. LIEB, Existence and uniqueness of the minimization solution of Choquard's nonlinear equation, Stud. Appl. Math. 57, 2 (1977), 93-105.

[3] P. L. Lions, The Choquard equation and related questions, Nonlinear Anal. 4, 6 (1980), 1063-1072.

[4] J. Mawhin And M. Willem, Critical point theory and Hamiltonian systems, Applied Mathematical Sciences 74, Springer-Verlag, New York, 1989.

[5] R. Penrose, On gravity's role in quantum state reduction, Gen. Relativ. Gravit. 28, 5 (1996), 581 600.

[6] Z. J. ZHANG, Multiple solutions of nonhomogeneous for related Choquard's equation, Acta Math. Sci. Ser. B Engl. Ed. 20, 3 (2000), 6-12.

[7] T. KÜPPER, Z. J. ZHAN AND H.Q. XIA, Multiple positive solutions and bifurcation for an equation related to Choquard's equation, Proc. Edinb. Math. Soc. (2) 46, 3 (2003), 597-607.

[8] N. ACKermann, On a periodic Schrödinger equation with nonlocal superlinear part, Math. Z. 248, 2 (2004), 423-443.

[9] Z. J. ZhANG, T. KÜPPER AND A. L. HU, H.-Q. XIA, Existence of a nontrivial solution for Choquard's equation, Acta Math. Sci. Ser. B Engl. Ed. 26, 3 (2006), 460-468.

[10] J. WeI AND M. WINTER, Strongly interacting bumps for the Schrödinger-Newton equations, J. Math. Phys. 50, 1 (2009), 012905, 22 pp.

[11] S. Cingolani, S. Secchi AND M. SQuassina, Semi-classical limit for Schrödinger equations with magnetic field and Hartree-type nonlinearities, Proc. Roy. Soc. Edinburgh Sect. A 140, 5(2010), 973-1009.

[12] L. MA AND L. ZHAO, Classification of positive solitary solutions of the nonlinear Choquard equation, Arch. Ration. Mech. Anal. 195, 2 (2010), 455-467.

[13] S. Cingolani, M. ClapP And S. SeCchi, Multiple solutions to a magnetic nonlinear Choquard equation, Z. Angew. Math. Phys. 63, 2 (2012), 233-248.

[14] S. Cingolani, M. ClapP And S. SeCChi, Intertwining semiclassical solutions to a SchrödingerNewton system, Discrete Contin. Dyn. Syst. Ser. S 6, 4 (2013), 891-908. 
[15] M. Clapp And D. SAlazar, Positive and sign chaning solutions to a nonlinear Choquard equation, J. Math. Anal. Appl. 407, 1 (2013), 1-15.

[16] V. Moroz And J. VAn Schaftingen, Ground states of nonlinear Choquard equations: existence, qualitative properties and decay asymptotics, J. Funct. Anal. 265, 2 (2013), 153-184.

[17] V. Moroz and J. VAn Schaftingen, Nonexistence and optimal decay of supersolutions to Choquard equations in exterior domains, J. Diff. Eqns. vol. 254, 8 (2013), 3089-3145.

[18] C. O. Alves AND M. B. YANG, Existence of semiclassical ground state solutions for a generalized Choquard equation, J. Diff. Eqns. vol. 257, 11 (2014), 4133-4164.

[19] D. F. LÜ, A note on Kirchhoff-type equations with Hartree-type nonlinearities, Nonlinear Anal. 99, 99 (2014), 35-48.

[20] S. Cingolani And S. Secchi, Semiclassical analysis for pseudorelativistic Hartree equations, J. Diff. Eqns. vol. 258, 12 (2015), 4156-4179.

[21] D. F. Lü, Existence and concentration of solutions for a nonlinear Choquard equation, Mediterr. J. Math., 12, 3 (2015), 839-850.

[22] V. Moroz And J. van Schaftingen, Groundstates of nonlinear Choquard equations: HardyLittlewood-Sobolev critical exponent, Commun. Contemp. Math., 17, 5 (2015), 1550005-1550017.

[23] V. Moroz and J. VAn Schaftingen, Semi-classical states for the Choquard equation, Calc. Var. Partial Differential Equations, 52, 1-2 (2015), 199-235. 